\title{
A 15-meter Multi-Gigabit W-band Bidirectional Wireless Bridge in Fiber-Optic Access Networks
}

Pang, Xiaodan; Vegas Olmos, Juan José; Lebedev, Alexander; Tafur Monroy, Idelfonso

Published in:

Proceedings of 2013 IEEE International Topical Meeting on Microwave Photonics

Link to article, DOI:

10.1109/mwp.2013.6724013

Publication date:

2013

Link back to DTU Orbit

Citation (APA):

Pang, X., Vegas Olmos, J. J., Lebedev, A., \& Tafur Monroy, I. (2013). A 15-meter Multi-Gigabit W-band Bidirectional Wireless Bridge in Fiber-Optic Access Networks. In Proceedings of 2013 IEEE International Topical Meeting on Microwave Photonics IEEE. https://doi.org/10.1109/mwp.2013.6724013

\section{General rights}

Copyright and moral rights for the publications made accessible in the public portal are retained by the authors and/or other copyright owners and it is a condition of accessing publications that users recognise and abide by the legal requirements associated with these rights.

- Users may download and print one copy of any publication from the public portal for the purpose of private study or research.

- You may not further distribute the material or use it for any profit-making activity or commercial gain

- You may freely distribute the URL identifying the publication in the public portal 


\title{
A 15-meter Multi-Gigabit W-band Bidirectional Wireless Bridge in Fiber-Optic Access Networks
}

\author{
Xiaodan Pang, J.J. Vegas Olmos*, Alexander Lebedev, Idelfonso Tafur Monroy \\ DTU Fotonik, Department of Photonics Engineering \\ Technical University of Denmark \\ Kgs. Lyngby, 2800, Denmark \\ *jjvo@fotonik.dtu.dk
}

\begin{abstract}
We present a bidirectional wireless bridge in the W-band enabling the seamless convergence between the wireless and fiber-optic access networks. In the downlink, a $16 \mathrm{Gbit} / \mathrm{s}$ QPSK signal is photonically up-converted at the wireless transmitter and electrically down-converted at the wireless receiver. The down-converted signal is re-modulated on to the lightwave and transmit further through the fiber-optic system. In the uplink, both up-and down-conversion are performed by electrical means. Furthermore, we investigate both passive and active wireless transmitters in this work for both downlink and uplink transmissions. With an active wireless transmitter, up to 15 meters wireless transmission is successfully achieved with a BER below the $7 \%$ FEC limit in the downlink.
\end{abstract}

Keywords - radio-over-fiber; w-band communications; optical access network

\section{INTRODUCTION}

Hybrid photonic-wireless systems are envisaged to seamlessly provide high-capacity connections over multiple scenarios, such as bridges, indoor distribution and distribution antenna systems, among others. Radio-over-fiber (RoF) techniques are supporting to bridge the gap between the large capacity of optical fibers and the flexibility of wireless transmission $[1,2]$. Currently, two main trends are impacting the RoF field: migration towards higher frequency bands and increase of the channel capacity. Frequency bands below $10 \mathrm{GHz}$ are already overcrowded, and therefore, open systems are looking for more spacious transmission bands such as $60 \mathrm{GHz}$ [3-5], 75-110 GHz [6-9] and even sub-THz [10]. The W-band $(75-110 \mathrm{GHz})$ is attractive because of its wide frequency bands, and seems to be an accepted good choice for transmission capacities in the order of multi-gigabits. The increase of the channel capacity is simultaneously impacting the field in the form of utilization of advanced modulation formats instead of the dominating approaches as on-off keying of the optical segment.

However, a main limitation of RoF systems is the poor conversion efficiency of the opto-electro-opto processes; namely, the efficiency of photodiodes and modulators. This efficiency shortcoming, in practical terms, introduces a limitation in the wireless range achieved by most experimental demonstrations of RoF systems, sometimes in the order of few centimeters or meters. This limitation may not affect scenarios such as short distance communications in data centers or

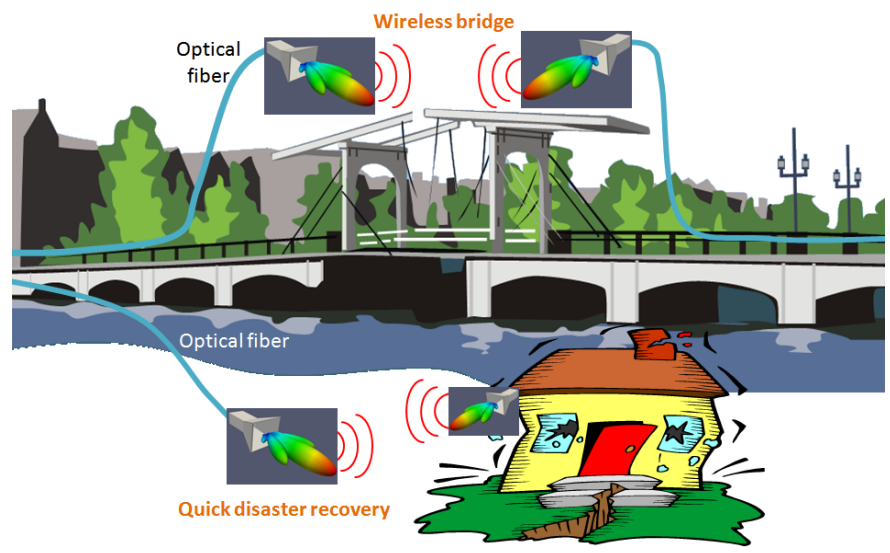

Fig. 1: Network scenarios based on hybrid photonic-wireless links, such as wireless bridges and quick disaster recovery systems.

indoor distributed antenna systems. However, scenarios such as disaster recovery, ad hoc last mile systems, or corporate communications as shown in Fig. 1, require transmission distances in the order of tens of meters.

In this paper, we show an active transmitter enabling wireless transmission up to 15 meters. The overall system consists of a bidirectional hybrid photonic-wireless link, comprising a fiber link, a wireless bridge, and a final fiber link. The paper is organized as follows: in Section II, we show the experimental setup of the proposed bidirectional fiber-wirelessfiber bridge. The experimental results for both passive and active wireless transmission are presented and discussed in Section III. Finally, conclusions are given in Section IV.

\section{EXPERIMENTAL SETUP}

Figure 2 presents the experimental setup of the proposed bidirectional wireless bridge for fiber-optic access systems. At the downlink (DL) transmitter, an continuous-wave (CW) lightwave at $1550.2 \mathrm{~nm}$ from an external cavity laser (ECL 1) is fed into a Mach-Zehnder modulator (MZM). By driving the MZM with a $20.35 \mathrm{GHz}$ modulation signal and adjusting the applied bias voltage to the modulator, the fourth order harmonics of the driving signal can be generated at the modulator output [11]. After the MZM placed an arrayed waveguide grating (AWG) with $50 \mathrm{GHz}$ channel grids, which is used to block the optical central carrier while separating the 


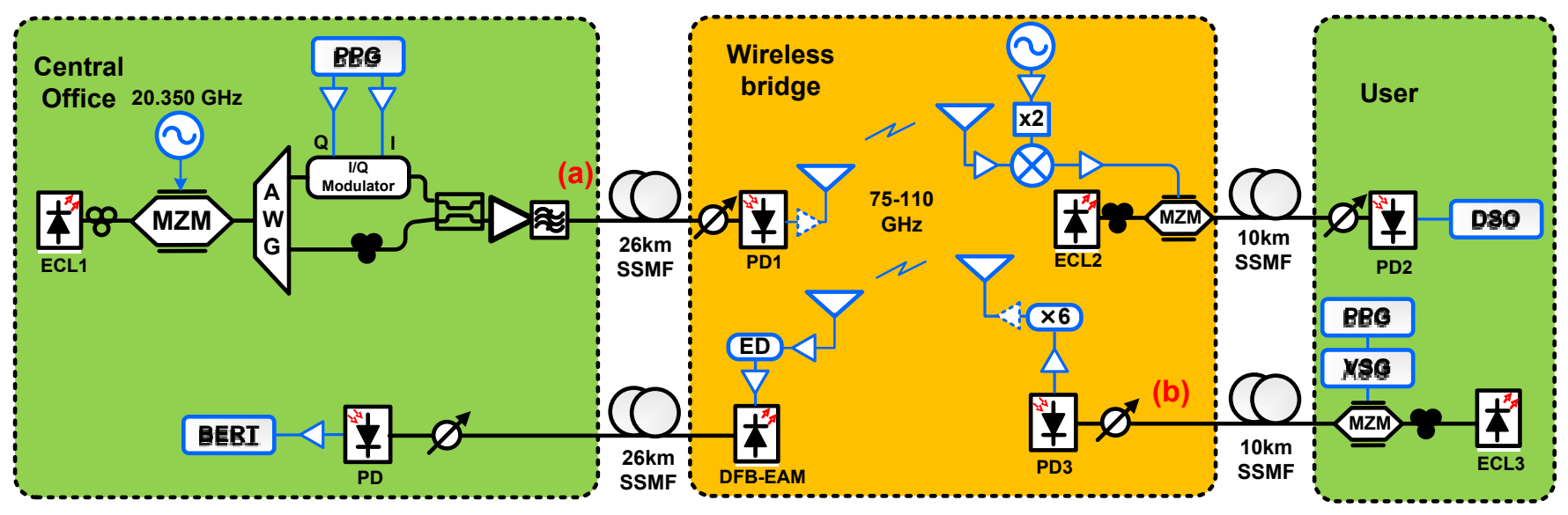

Fig. 2: Experimental setup. ECL: external cavity laser. MZM: Mach-Zehnder modulator. AWG: arrayed waveguide grating. PPG: pulse pattern generator. PD: photodetector. DSO: digital storage scope. VSG: vector signal generator. ED: envelope detector. DFB-EAM: distributed feedback laser - electroabsorption modulator. BERT: bit-error-rate tester.
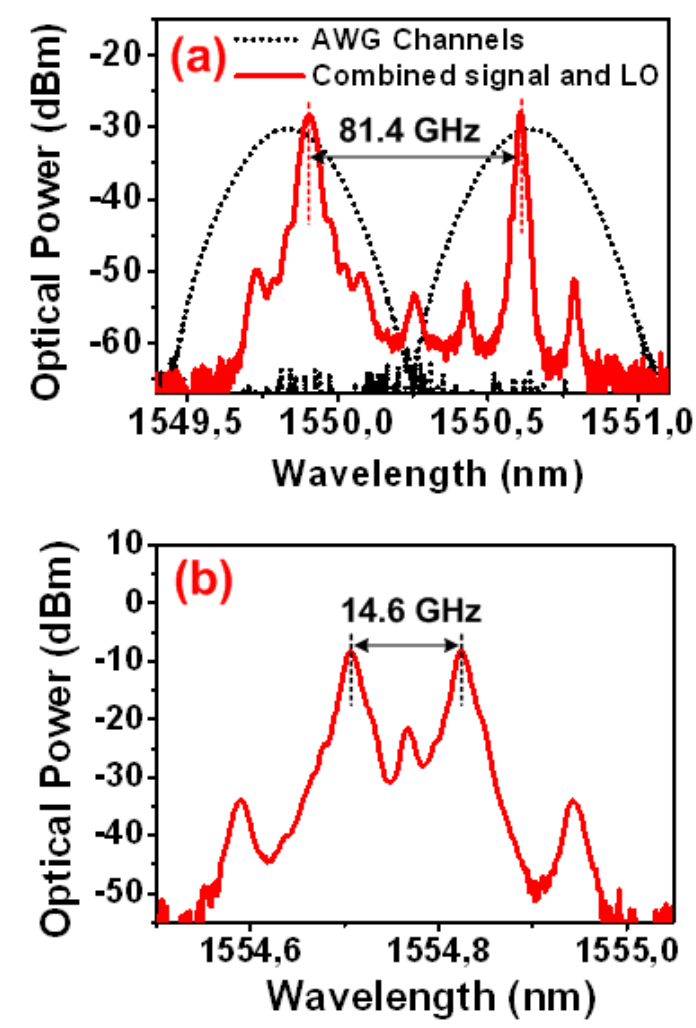

Fig. 3: Optical spectra measured at downlink point (a) and uplink point (b) in Fig. 2.

upper / lower sidebands (USB / LSB). The frequency separation between the USB and LSB is $81.4 \mathrm{GHz}$. The LSB is launched into a integrated $\mathrm{LiNbO} 3$ dual-parallel MZM driven by a two-channel 8 Gbaud pseudo-random binary sequences (PRBS) with a word length of $2^{15}-1$, resulting in an overall $16 \mathrm{Gbit} / \mathrm{s}$ QPSK signal at the output. On the other hand, the USB is polarization aligned and combined with the LSB at a $3 \mathrm{~dB}$ coupler, acting as a carrier generating local oscillator (LO) later for the optical heterodyne up-conversion. The optical spectra of the AWG channels and the combined signal is shown in Fig. 3 (a). At the output of the DL transmitter, the combined signal and LO forming a single side-band (SSB) RoF signal is launched into a $26 \mathrm{~km}$ standard single mode fiber (SSMF). After transmission, the signal is up-converted to a $\mathrm{W}$ band RF signal at a $100 \mathrm{GHz}$ photodetector. (PD 1). In this experiment, we test wireless transmissions both with and without a W-band power amplifier (PA) before radiating the signal to the air, representing the active and the passive wireless transmitter cases. A wireless bridge is established between a pair of $25 \mathrm{dBi}$ horn antennas. At the receiver, the received signal is firstly amplified by a $40 \mathrm{~dB}$ gain low noise amplifier (LNA), before being electrically down-converted to a intermediate frequency (IF) centered at $6.4 \mathrm{GHz}$ at a $\mathrm{W}$-band balanced mixer. The IF signal is then re-modulated onto lightwave at a second MZM and transmitted a final $10 \mathrm{~km}$ SSMF. At the receiver, the IF-over-fiber signal is converted back to electrical domain at a second PD and sampled by a $40 \mathrm{GSa} / \mathrm{s}$ digital storage oscilloscope (DSO) for offline signal processing and demodulation.

In the uplink (UL) direction, a $1.25 \mathrm{Gbit} / \mathrm{s}$ electrical signal from a pulse pattern generator (PPG) is firstly up-converted to a IF at $7.3 \mathrm{GHz}$ by a vector signal generator (VSG). Then the IF signal is modulated at the minimum transmission point of a MZM, forming a carrier suppressed-double sideband (CS-DSB) IF-over-fiber signal, the optical spectrum of which is shown in Fig. 3 (b). The signal transmits over the same $10 \mathrm{~km} \mathrm{SSMF} \mathrm{as}$ used in the DL before being converted back to electrical at a PD. After that the IF signal is fed to an electrical frequency sextupler, which up-converts the signal to an $81.6 \mathrm{GHz}$ amplitude shifted-keying (ASK). Detailed characterization of the frequency sextupler can be found in [12]. Similarly, both the active and passive wireless transmitters are tested, by adding or skipping the $\mathrm{W}$-band PA. The signal is then radiated by a $24 \mathrm{dBi}$ horn antenna. At the wireless receiver, the signal is picked up by an identical antenna and then fed to a zero-biased Schottky diode performing a function of an envelope detector (ED) down-converting the $\mathrm{W}$-band signal directly to baseband. 

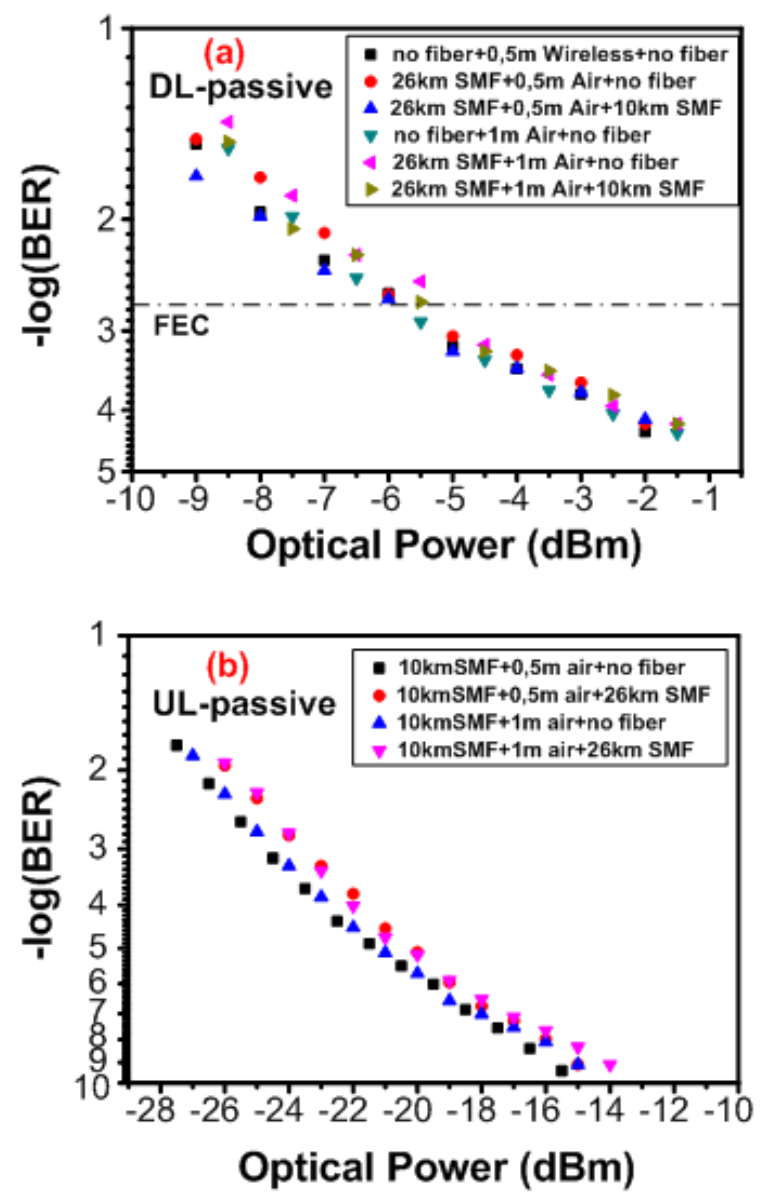

Fig. 4: Measured BER vs. received optical power in the (a) DL and (b) UL, with passive wireless transmitter.

The down-converted signal is directly modulated onto lightwave at an distributed feedback laser - electroabsorption modulator (DFB-EAM). After transmitting the same $26 \mathrm{~km}$ SSMF fiber link as the DL, the signal is recovered by a $10 \mathrm{G}$ $\mathrm{PD}$ and the transmission is evaluated in real time by a bit-errorrate tester (BERT).

\section{RESULTS AND DISCUSSION}

\section{A. Passive wireless transmitter}

The transmission performance of the system is experimentally evaluated by means of bit-error-rates (BERs). In the first step, we skip the W-band PA at the transmitter in both DL and UL directions, benchmarking the transmission performance of the passive wireless transmission case. Figure 4(a) and (b) shows the measured BER curves in both DL and UL in such case. We evaluate the BERs as a function of the received optical power at PD after the last piece of fiber transmission. The wireless power in both directions are adjusted to give the best performance for each wireless distance during the measurements, which means maximizing the signal-to-noise ratio (SNR), while minimizing the signal distortion caused by the LNA saturation at the wireless receiver.
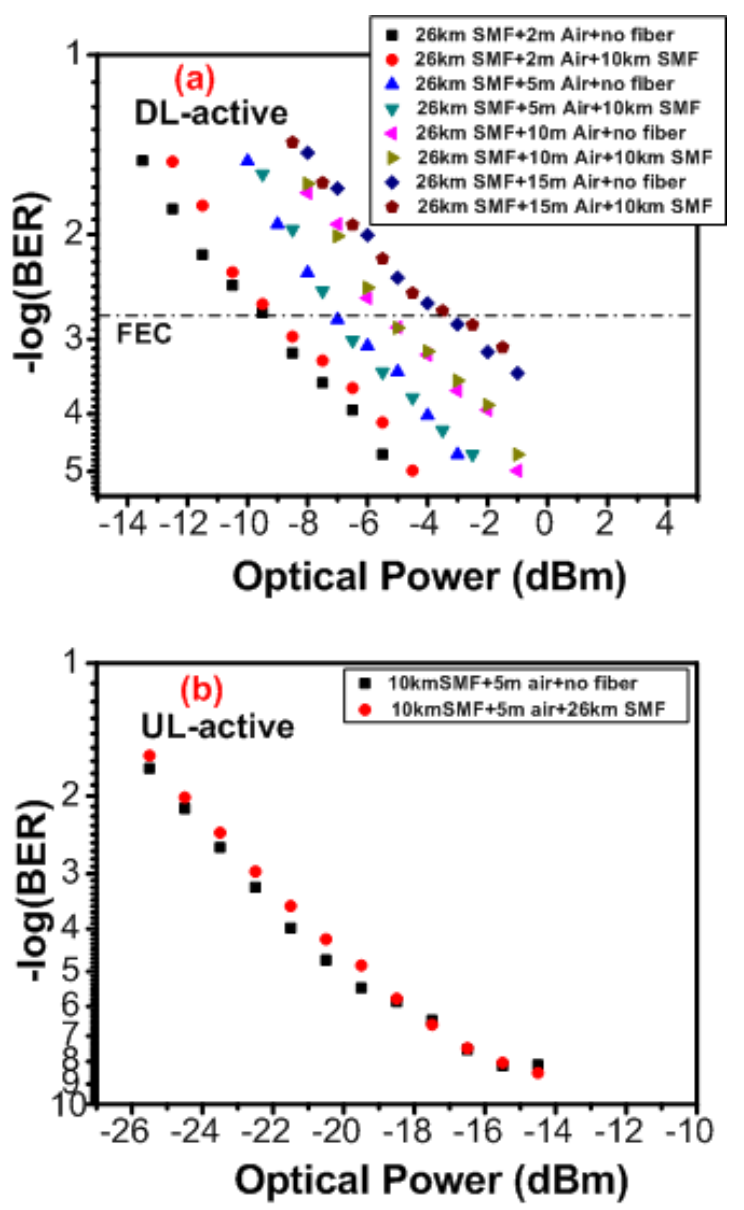

Fig. 5: Measured BER vs. received optical power in the (a) DL and (b) UL, with active wireless transmitter.

In the DL, as shown in Fig. 4(a), the received QPSK signal after the compounded $36 \mathrm{~km}$ SSMF plus the wireless bridge can be recovered within the 7\% forward error correction (FEC) limit of $2 \times 10^{-3}$. Due to the low radiated RF power in this passive case, the transmitted air distances of the wireless bridge are limited to $1 \mathrm{~m}$. The receiver sensitivity at the FEC limit is $5.5 \mathrm{dBm}$ and $-6 \mathrm{dBm}$ for $0.5 \mathrm{~m}$ and $1 \mathrm{~m}$ wireless transmission without fiber links, respectively. Negligible penalty $(<0.5 \mathrm{~dB})$ is observed after transmission over both the first $26 \mathrm{~km}$ and the final $10 \mathrm{~km}$ fiber links. For the UL, Error free $\left(B E R<10^{-9}\right)$ transmission can be achieved in all tested cases. The receiver sensitivity at BER of $10^{-9}$ is $-15.5 \mathrm{dBm}$ and $-15 \mathrm{dBm}$ for $0.5 \mathrm{~m}$ and $1 \mathrm{~m}$ wireless transmission with only the first $10 \mathrm{~km}$ SSMF fiber link. $\sim 1 \mathrm{~dB}$ penalty is introduced by the final $26 \mathrm{~km}$ SSMF transmission, which can also be regarded as negligible.

\section{B. Active wireless transmitter}

After benchmarking the passive wireless transmission case, we add a W-band PA of $14 \mathrm{~dB}$ gain to the wireless transmitter to boost the radiated RF power. By doing this the wireless coverage is highly increased. In the DL direction, we succeeded reaching up to 15 meters wireless transmission with received BER performance within the 7\% FEC limit. Figure 5(a) presents the BER performances of the DL 


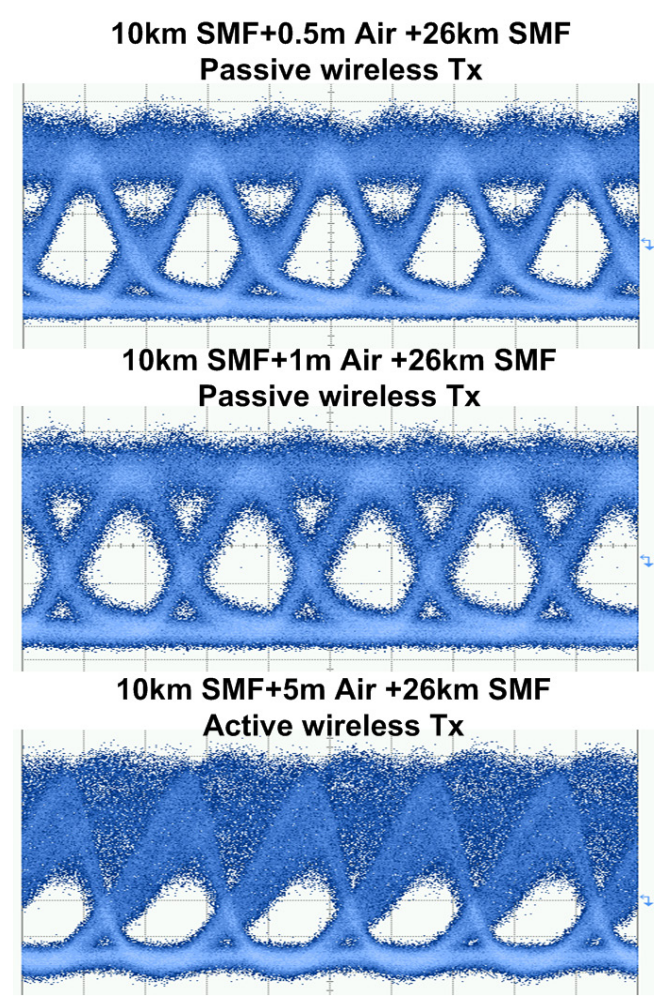

Fig. 6: Received UL signal eye diagrams at error free $\left(\mathrm{BER}<10^{-9}\right)$ transmission points.

transmission with wireless distance of $2 \mathrm{~m}, 5 \mathrm{~m}, 10 \mathrm{~m}$ and $15 \mathrm{~m}$. With only the first $26 \mathrm{~km} \mathrm{SSMF}$ and the wireless link, the receiver sensitivities at the FEC limit are $-9.5 \mathrm{dBm},-7 \mathrm{dBm}$, $-5 \mathrm{dBm}$ and $-3 \mathrm{dBm}$. These performance differences are due to the different SNR at the receiver for different wireless distances. The final $10 \mathrm{~km}$ SSMF transmission induces $<0.5 \mathrm{~dB}$ penalty in all wireless distances. It is noted that longer transmission distances are limited by the frequency selective fading induced by multipath effect in the indoor experimental environment rather than wireless power. Therefore further efforts to mitigate this issue will be necessary.

In the UL direction, the transmission demonstration in the active case is limited to 5 meters due to the physical constrains of the laboratory condition. According to the measured BER curves shown in Fig. 5(b), BER of $10^{-9}$ can be reached with received power larger than $-14 \mathrm{dBm}$, despite a slight error floor is observed due to the slightly distortion induced by the $\mathrm{W}$ band PA. Again, no penalty of the sensitivity is induced after the final $26 \mathrm{~km}$ SSMF transmission. The received eye diagrams after the total $36 \mathrm{~km} \mathrm{SSMF}$ and $0.5 \mathrm{~m} / 1 \mathrm{~m}$ air transmission in the passive case and $5 \mathrm{~m}$ transmission in the active case are shown in Fig. 6. We can see that in the passive case the eye diagrams are clear and open without being distorted, while after adding the $\mathrm{W}$-band PA in the active wireless transmitter case, a certain level of distortion in the eye diagram can be observed. In spite of certain degradation induced by the active transmitter, achieving transmission over long distance of up to 15 meters can be expected, as the wireless signal has a large enough power margin. Moreover, the UL signal is more immune to frequency selective fading in the indoor environment due to its narrower bandwidth compared with the DL signal.

\section{CONCLUSIONS}

This paper has shown an experimental demonstration of a bidirectional hybrid photonic-wireless link with a $81.4 \mathrm{GHz}$ Wband wireless transmission. Two different transmitters are shown, passive and active, the latter enabling a wireless transmission of 15 meters. The fiber guided sections compounded a total of $36 \mathrm{~km}$. The demonstrated channel capacity is $16 \mathrm{Gbit} / \mathrm{s}$, although higher capacity can be achieved since the W-band has more available bandwidth. Both the downlink and the uplink reach error free performance without perceptible error flour, which indicates that the capacity higher bound is yet to be reached.

\section{ACKNOWLEDGMENT}

J.J. Vegas Olmos acknowledges the Marie Curie program for partly funding this research through the WISCON project.

\section{REFERENCES}

[1] T. Nagatsuma et al., "Millimeter- and THz-wave Photonics Towards 100-Gbit/s Wireless Transmission," Proc. 23rd Annu. Meeting IEEE Photon. Soc., We4 2010.

[2] JJ Vegas Olmos et al., "Reconfigurable radio-over-fiber networks: Multiple-access functionality directly over the optical layer," IEEE Trans. Microw. Theory Techn., vol. 58, pp. 3001-3010, 2010.

[3] M. Weiss et al., "27 Gbit/s photonic wireless $60 \mathrm{GHz}$ transmission system using 16-QAM OFDM," Proc. MWP'09, PDP, 2009.

[4] W. Jiang et al., "40 Gb/s RoF Signal Transmission with $10 \mathrm{~m}$ Wireless Distance at $60 \mathrm{GHz}$," in OFC/NFOEC 2012, paper OTu2H.1.

[5] C. Ho et al., "50-Gb/s Radio-over-Fiber System Employing MIMO and OFDM Modulation at $60 \mathrm{GHz}$," in OFC/NFOEC 2012, paper OM2B.3.

[6] A. Kanno et al., "40 Gb/s W-band (75-110 GHz) 16-QAM radio-overfiber signal generation and its wireless transmission," Opt. Exp., vol. 19, pp. B56-B63, Dec. 2011.

[7] L. Deng et al., "42.13 Gbit/s 16qam-OFDM photonics-wireless transmission in 75-110 GHz band," Progress In Electromagnetics Research, vol. 126, pp. 449-461, 2012.

[8] X. Pang et al., "100 Gbit/s hybrid optical fiber-wireless link in the Wband (75-110 GHz)," Opt. Exp., vol. 19, pp. 24944-24949, Dec. 2011.

[9] J. Zhang et al., "Multichannel 120-Gb/s Data Transmission Over 2 x 2 MIMO Fiber-Wireless Link at W-Band," IEEE Photon. Technol. Lett., vol.25, no.8, pp.780,783, 2013

[10] S. Koenig et al., "100 Gbit/s Wireless Link with mm-Wave Photonics," in $O F C / N F O E C$ 2013, paper PDP5B.4.

[11] H. Kiuchi et al., "High Extinction Ratio Mach-Zehnder Modulator Applied to a Highly Stable Optical Signal Generator," IEEE Trans. Microw. Theory Techn., vol.55, pp.1964, 2007.

[12] X. Pang et al., "Experimental characterization of a hybrid fiber-wireless transmission link in the 75 to $110 \mathrm{GHz}$ band," Opt. Eng., vol. 51, pp. 045004-1-045004-5, 2012. 